\title{
Possible New Role of Vascular Endothelial Growth Factor-D During the Acute Phase of Kawasaki Disease
}

\author{
Keiichi Hirono, MD, PhD; Fukiko Ichida, MD, PhD
}

$\mathbf{K}$ awasaki Disease (KD) was first reported in 1967, yet the cause has not been established. A relationship between KD and vascular endothelial growth factor (VEGF) was initially established 13 years ago. ${ }^{1-3}$ In this issue of the Journal, Ebata et al describe a role for VEGF-D during the acute stage of $\mathrm{KD}$ and report that its expression increased during lymphangiogenesis. ${ }^{4}$ Here we review the role of VEGF in KD and its possible role during the acute phase of $\mathrm{KD}$, especially in the development of coronary artery lesions (CAL).

\section{Article p 1455}

$\mathrm{KD}$ is the most common systemic vasculitis syndrome primarily affecting small and medium sized arteries, particularly the coronary artery. Although timely treatment with high-dose intravenous immune globulin (IVIG) is now accepted as reducing the incidence of CAL, approximately $15 \%$ of patients do not respond to IVIG treatment and are at the highest risk for developing CAL.

The etiologic nature of KD and the pathogenesis of CAL largely remain to be determined. Previous histopathologic studies revealed that early coronary vascular lesions are infiltrated by a large number of mononuclear cells, such as macrophages and lymphocytes, leading to the assumption that these cells play a key role in the progression of CAL. In acute $\mathrm{KD}$, the migration and proliferation of endothelial cells have been demonstrated to be markedly enhanced by cytokines. As a matter of fact, during the acute phase of KD, serum levels of proinflammatory cytokines are elevated..$^{5}$ In addition to these proinflammatory cytokines, expression of VEGF, and of markers of local inflammation, including the family of damage-associated molecular pattern molecules, such as myeloidrelated protein (MRP) 8/MRP14 and S100A12, which respond to initiation of the innate immune response, increases during acute KD and plays a crucial role in inflammation. ${ }^{3,6,7}$

VEGF and their endothelial tyrosine kinase receptors are central regulators of vasculogenesis, angiogenesis and lymphangiogenesis. VEGF is expressed by a variety of cell types, including aortic smooth muscle cells, macrophages, and myocytes. In addition, lymphocytes, neutrophils, and platelets have recently been shown to express VEGF. Currently, 7 members in the VEGF family have been identified: VEGF-A, -B, -C, -D, -E (viral VEGF analogs), -F (snake venom VEGFs), and placental growth factor. VEGFs belong to the plateletderived growth factor/VEGF superfamily of secreted dimeric glycoprotein growth factors that contain a cysteine knot motif consisting of 8 regularly spaced cysteine residues. The VEGF ligands bind with differing specificities to 3 , mostly endothelial transmembrane tyrosine kinase, receptors: VEGFR-1/ fms-like tyrosine kinase (Flt) 1, VEGFR-2/fuman kinase insert domain receptor/mouse fetal liver kinase 1 and VEGFR-3/ Flt 4 (Figure). All VEGFRs have a conserved intracellular split tyrosine kinase domain and a series of extracellular Ig-like domains. Neuropilins-1 and -2, originally identified as semaphoring receptors, function as coreceptors for specific VEGFs. They share a common structure, yet they bind VEGFs with distinct affinities and specificities.

VEGF-D (also known as $c$-fos-induced growth factor) is secreted into the extracellular space as a full-length VEGF-D homodimer. After secretion, proprotein convertases cleave the $\mathrm{C}$ - and N-terminal propeptides from the VEGF-homology domain to form the mature VEGF-D, which binds to VEGFR-2 and -3 with higher affinities than the full-length unprocessed VEGF-D (Figure). However, as VEGF-D seems to be largely dispensable for the development of the lymphatic system, its physiological role remains unclear. Vascular endothelial cells express VEGFR-2, and its expression is upregulated inter alia by angiogenesis. VEGF-D exerts angiogenic effects on binding to VEGFR-2, whereas when bound to VEGFR-3 on lymphatic endothelium it stimulates lymphangiogenesis (Figure). VEGFR-3 is initially widely expressed in endothelial cells during murine embryogenesis, but later in development its expression becomes largely restricted to the lymphatic endothelium. ${ }^{8}$ In adult human tissues, VEGFR-3 is specific to the lymphatic endothelium, with the exception of some fenestrated and discontinuous blood capillary bed. ${ }^{9}$ VEGFR-3 phosphorylation has been shown to lead to PI3Kdependent Akt activation and PKC-dependent activation of the $\mathrm{p} 42 / \mathrm{p} 44$ MAPK pathway. ${ }^{10}$

It has been suggested in several studies ${ }^{1-3}$ that VEGF has a key role in the deterioration of vasculitis in the acute phase of KD. The studies have shown that serum and plasma levels of VEGF are significantly elevated in both the acute and subacute phases of KD and that circulating levels could be associated with the risk of developing CAL. It has been hypothesized that the high levels of VEGF could contribute to the pathologic findings observed in vascular tissue in $\mathrm{KD}$,

The opinions expressed in this article are not necessarily those of the editors or of the Japanese Circulation Society.

Received April 17, 2011; accepted April 18, 2011; released online April 29, 2011

Department of Pediatrics, Graduate School of Medicine, University of Toyama, Toyama (K.H., F.I.), Japan; Division of Cardiology, The

Heart Institute, Cincinnati Children's Hospital Medical Center, Cincinnati, OH (K.H.), USA

Mailing address: Fukiko Ichida, MD, PhD, Department of Pediatrics, Graduate School of Medicine, University of Toyama, 2630 Sugitani,

Toyama 930-0194, Japan. E-mail: fukiko@med.u-toyama.ac.jp

ISSN-1346-9843 doi:10.1253/circj.CJ-11-0408

All rights are reserved to the Japanese Circulation Society. For permissions, please e-mail: cj@j-circ.or.jp 


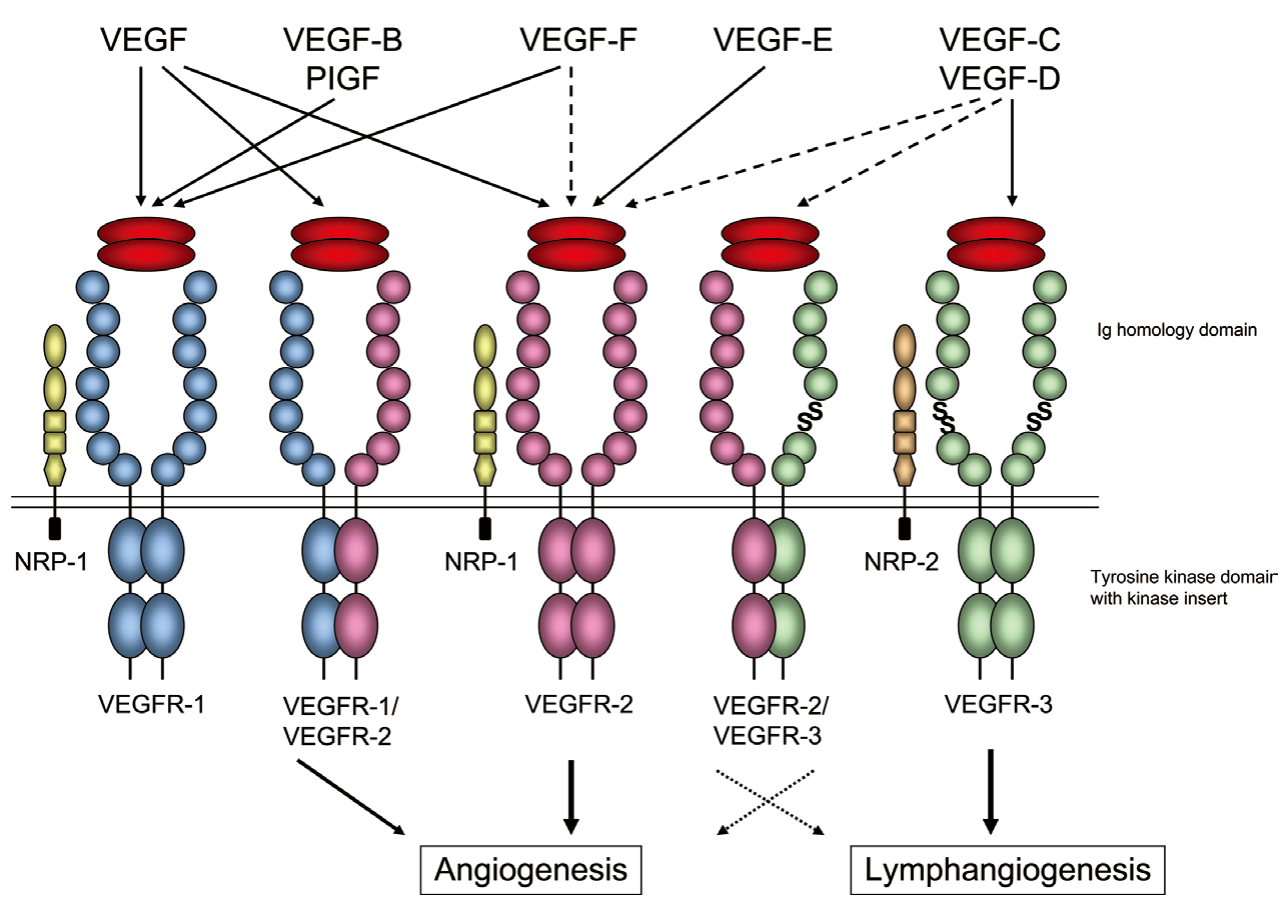

Figure. The vascular endothelial growth factor (VEGF) family ligands and receptors. VEGF, VEGF-B and placental growth factor (PIGF) bind VEGFR-1 and VEGFR-2 on the vascular endothelium. VEGF-C and VEGF-D primarily bind VEGFR-3 on lymphatic endothelium.

such as subendothelial edema, gap formation, and fenestrations of endothelial cells. ${ }^{11}$ Several single-nucleotide polymorphisms in the $V E G F$ gene have been found to be associated with differences in VEGF protein production. ${ }^{12}$

There have been many studies reporting a role for VEGF in $\mathrm{KD}$, but the study by Ebata et al represents the first report of a relationship between VEGF-D and KD. They conclude that serum VEGF-D levels increased after IVIG therapy in patients with acute KD and were significantly lower in patients with CAL compared to those without CAL. They also showed that the cross-sectional areas of lymphatic vessels in cardiac tissues were enlarged in patients with acute KD while VEGF-D protein was detected on the endothelium of the enlarged lymphatic vessels. Lymphatic vessels are thought to be maintained in a collapsed state and are important for the drainage of interstitial fluid under pathological conditions. ${ }^{13}$ Therefore, these new findings might reflect disruption or insufficiency of the regulation of lymphangiogenesis mediated by VEGF-D during the acute stage of KD.

Although further studies are required to elucidate the mechanism of how VEGF-D affects the outcome of KD, the evidence so far suggests that VEGF-D plays an important role and may serve as a biomarker for $\mathrm{KD}$, as well as providing a novel therapeutic target.

\section{References}

1. Maeno N, Takei S, Masuda K, Akaike H, Matsuo K, Kitajima I, et al. Increased serum levels of vascular endothelial growth factor in Kawasaki disease. Pediatr Res 1998; 44: 596-599.

2. Terai M, Yasukawa K, Narumoto S, Tateno S, Oana S, Kohno Y. Vascular endothelial growth factor in acute Kawasaki disease. $\mathrm{Am}$ J Cardiol 1999; 83: 337-339.

3. Hamamichi Y, Ichida F, Yu X, Hirono KI, Uese KI, Hashimoto I, et al. Neutrophils and mononuclear cells express vascular endothelial growth factor in acute Kawasaki disease: Its possible role in progression of coronary artery lesions. Pediatr Res 2001; 49: 74-80.

4. Ebata R, Abe J, Yasukawa K, Hamada H, Higashi K, Suwazono Y, et al. Increased production of vascular endothelial growth factor-D and lymphangiogenesis in acute Kawasaki disease. Circ J 2011; 75: $1455-1462$.

5. Sakata K, Hamaoka K, Ozawa S, Niboshi A, Yahata T, Fujii M, et al. Matrix metalloproteinase-9 in vascular lesions and endothelial regulation in Kawasaki disease. Circ J 2010; 74: 1670-1675.

6. Hirono K, Foell D, Xing Y, Miyagawa-Tomita S, Ye F, Ahlmann M, et al. Expression of myeloid-related protein- 8 and -14 in patients with acute Kawasaki disease. J Am Coll Cardiol 2006; 48: 1257-1264.

7. Ye F, Foell D, Hirono KI, Vogl T, Rui C, Yu X, et al. Neutrophilderived S100A12 is profoundly upregulated in the early stage of acute Kawasaki disease. Am J Cardiol 2004; 94: 840-844.

8. Kaipainen A, Korhonen J, Mustonen T, van Hinsbergh VW, Fang $\mathrm{GH}$, Dumont D, et al. Expression of the fms-like tyrosine kinase 4 gene becomes restricted to lymphatic endothelium during development. Proc Natl Acad Sci USA 1995; 92: 3566-3570.

9. Partanen TA, Arola J, Saaristo A, Jussila L, Ora A, Miettinen M, et al. VEGF-C and VEGF-D expression in neuroendocrine cells and their receptor, VEGFR-3, in fenestrated blood vessels in human tissues. FASEB J 2000; 14: 2087-2096.

10. Makinen T, Veikkola T, Mustjoki S, Karpanen T, Catimel B, Nice $\mathrm{EC}$, et al. Isolated lymphatic endothelial cells transduce growth, survival and migratory signals via the VEGF-C/D receptor VEGFR-3. EMBO J 2001; 20: 4762-4773.

11. Yasukawa K, Terai M, Shulman ST, Toyozaki T, Yajima S, Kohno $\mathrm{Y}$, et al. Systemic production of vascular endothelial growth factor and fms-like tyrosine kinase-1 receptor in acute Kawasaki disease. Circulation 2002; 105: 766-769.

12. Kariyazono H, Ohno T, Khajoee V, Ihara K, Kusuhara K, Kinukawa $\mathrm{N}$, et al. Association of vascular endothelial growth factor (VEGF) and VEGF receptor gene polymorphisms with coronary artery lesions of Kawasaki disease. Pediatr Res 2004; 56: 953-959.

13. Nagy JA, Vasile E, Feng D, Sundberg C, Brown LF, Detmar MJ, et al. Vascular permeability factor/vascular endothelial growth factor induces lymphangiogenesis as well as angiogenesis. J Exp Med 2002; 196: 1497-1506. 